\title{
Berliner Orientalismus / orientalisches Berlin. Kulturkritik als Diskurskritik in den Briefen aus Berlin von Heine, Kerr und Lasker-Schüler
}

Orientalisme berlinois / Berlin oriental. Critique du discours journalistique et critique de la culture dans les Briefe aus Berlin de Heine, Kerr et Lasker-

Schüler

Berlin Orientalism / oriental Berlin. Cultural Criticism as Discourse Criticism in Briefe aus Berlin from Heine, Kerr and Lasker-Schüler

\section{Sibylle Schönborn}

\section{(2) OpenEdition}

\section{Édition électronique}

URL : http://journals.openedition.org/ceg/656

DOI : $10.4000 /$ ceg. 656

ISSN : 2605-8359

\section{Éditeur}

Presses Universitaires de Provence

\section{Édition imprimée}

Date de publication : 18 novembre 2016

Pagination : 139-149

ISBN : 979-10-320-0087-8

ISSN : 0751-4239

\section{Référence électronique}

Sibylle Schönborn, « Berliner Orientalismus / orientalisches Berlin. Kulturkritik als Diskurskritik in den Briefen aus Berlin von Heine, Kerr und Lasker-Schüler », Cahiers d'Études Germaniques [Online], 71 | 2016, Online erschienen am: 18 Mai 2018, abgerufen am 27 November 2020. URL : http:// journals.openedition.org/ceg/656 ; DOI : https://doi.org/10.4000/ceg.656 


\title{
Berliner Orientalismus / orientalisches Berlin Kulturkritik als Diskurskritik in den Briefen aus Berlin von Heine, Kerr und Lasker-Schüler
}

\author{
Sibylle SCHÖNBORN \\ Universität Düsseldorf
}

In den großen Tageszeitungen setzt sich um 1900 eine Textsorte mit einer bis ins 18. Jahrhundert zurückweisenden Geschichte durch, die ihren Ort an der Schnittstelle zwischen politischem Teil und Feuilleton, zwischen Fakten und Fiktionen, zwischen Bericht, Kommentar und Erzählung findet, die so genannten „Briefe aus ***.. Im regelmäßigen Rhythmus vermitteln die Schreiber in ihren Briefen aus den Metropolen Europas wie London, Paris, Wien und Berlin zwischen den Großstädten oder berichten aus dem Zentrum in die Provinz. Ein Kennzeichen dieser Korrespondentenberichte besteht in ihrer Autoreflexivität, so dass die Sparte des Feuilletons einmal als „Nachrichtenschreiberei“ order unsystematische „Assoziazion der Ideen“", ein anderes Mal als private Plauderei begriffen wird. Heinrich Heines Briefe aus Berlin für den Rheinisch-Westfälischen Anzeiger aus dem Jahr 1822 stellen so etwas wie das Urmuster dieser journalistischen Form zwischen faktualem Bericht, literarischer Fiktion und kritischer Reflexion für die Moderne dar, an dem sich seine Nachfolger messen oder wie Karl Kraus in seinem berühmten Aufsatz Heine und die Folgen ${ }^{2}$ kritisch abarbeiten. Eben jener Karl Kraus verfasst neben seiner Kritik des Feuilletonismus' aber selbst zwischen 1896 und 1897 Briefe aus Wien³ für die Hauptstadt Schlesiens in der Breslauer Zeitung. Während sein Kollege und erbitterter Kontrahent, Alfred Kerr, wie vor ihm Heinrich Hart ${ }^{4}$ zwischen 1895 und 1900 für die dieselbe Zeitung aus Berlin ${ }^{5}$ berichtet. Heines, Harts, Kerrs oder auch Joseph Roths spätere

1. Heinrich Heine, Briefe aus Berlin I, in ders., Historisch-kritische Gesamtausgabe der Werke (DHA), hrsg. v. Manfred Windfuhr, Bd. 6, Briefe aus Berlin, Über Polen, Reisebilder I/II (Prosa), bearb. von Jost Hermand, Hamburg, Hoffmann und Campe, 1973, S. 7-53, hier S. 9.

2. Karl Kraus, „Heine und die Folgen“, in Die Fackel 13 (August 1911), Nr. 329/330, S. 7.

3. Karl Kraus, Frühe Schriften 1892-1900, hrsg. v. Joh. J. Braakenburg, 2 Bde., München, Kösel, 1979.

4. Heinrich Hart, Mongolenhorden im Zoologischen Garten. Berliner Briefe, hrsg. von Lars-Broder Keil, Berlin, Aufbau-Verlag, 2005.

5. Alfred Kerr, Wo liegt Berlin. Briefe aus der Reichshauptstadt 1895-1900, hrsg. v. Günther Rühle. Berlin, Aufbau-Verlag, 1997. 
Briefe aus Berlin 6 für die Frankfurter Zeitung haben diesem Genre nicht nur innerhalb der literarischen Gattung der Kleinen Form einen festen Platz und ein unverwechselbares Gesicht verliehen, sondern zugleich eine literarische Form des Kommentars und der Kritik etabliert, mit der sie die Gesellschaft und Kultur ihrer Zeit in den großen Zentren Europas beobachten. Eine Gattungsgeschichte dieser spannenden Kleinen Form der Kulturkritik im Medium der Zeitung oder Zeitschrift steht bisher noch aus. Im Folgenden soll es weniger um eine inhaltlich-thematische Auseinandersetzung mit diesen Texten, als vielmehr um die Frage nach ihrer Aneignung der traditionellen Gattung des Briefs als öffentlich-literarische Form im neuen medialen Kontext der Tageszeitung gehen.

Dass sich der Brief im Feuilleton als erfolgreiches Format diesen herausragenden Platz erobern konnte, liegt u.a. in seiner Vertrautheit für den Leser als Gebrauchsform subjektiv-privater bis intimer Kommunikation begründet, die der Korrespondentenbericht im Feuilleton zum einen mit der persönlichen Adressierung an seine Leser nachahmt und zum anderen für einen offenen dialogischen Meinungsaustausch im kritischen, kulturräsonierenden Diskurs nach Jürgen Habermas' Strukturwandel der Öffentlichkeit ${ }^{7}$ umformt. Diese konstitutiven Merkmale des Briefs korrespondieren in idealer Weise mit der hybriden Form des Feuilletons als kulturkritische Plauderei zwischen Literatur, Reflexion und Kritik in der Tagespresse wie sie Hildegard Kernmayer beschrieben hat: „Und auch gattungspoetologisch besehen, firmiert das Feuilleton, hier verstanden als Textsorte und als Rubrik, als „Hybrid', der seine spezifische Gestalt in Zusammenführung unterschiedlicher literarischer Textfunktionen und Formtraditionen ausbildet." ${ }^{8}$ Die Geschichte des Briefs als kulturkritisches Format im Feuilleton soll im Folgenden exemplarisch an einer sich von Heine bis zu Lasker-Schüler durchziehenden Kritik des deutschen Orientalismusdiskurses ${ }^{9}$ im Sinne Edward Saids nachvollzogen und an diesem Beispiel kulturkritischen Raisonnements das selbstreflexive Spiel mit der Gattung des Briefs in den Berliner Briefen von Heine, Kerr und Lasker-Schüler nachvollzogen werden.

\section{Heinrich Heines Briefe aus Berlin 1822}

Heine eröffnet seinen ersten Brief für den Rheinisch-Westfälischen Anzeiger vom 26. Januar 1822 - ganz gemäß den Regeln zur Abfassung eines guten Briefs -

6. Joseph Roth, Werke, Bd. I und 2. Das journalistische Werk, hrsg. von Hermann Kesten, Köln, Kiepenheuer \&t Witsch, 1990. Vgl. dazu: Telse Hartmann, „Szenarien der Deplatzierung in Joseph Roths Berlindiskurs“, in Stéphane Pesnel / Erika Tunner et al. (Hrsg.), Joseph Roth - Städtebilder, Zur Poetik, Philologie und Interpretation von Stadtdarstellungen in den 1920er und 1930er Jahren, Schriftenreihe der Internationalen Joseph Roth Gesellschaft, Berlin, Frank \& Timme, 2015, S. 101-118.

7. Jürgen Habermas, Strukturwandel der Öffentlichkeit. Untersuchen zu einer Kategorie der bürgerlichen Gesellschaft, Berlin und Neuwied, Luchterhand, 1962, S. 112-172.

8. Hildegard Kernmayer, „Sprachspiel nach besonderen Regeln. Zur Gattungspoetik des Feuilletons“, in Zeitschrift für Germanistik, Neue Folge XXII, 3/2012, S. 511.

9. Edward W. Said, Orientalism, New York, Pantheon Books, 1978. S. 4-73. 
mit einer privaten Adresse an den Herausgeber der Zeitung, der stellvertretend für das im zweiten Abschnitt angesprochene Lesepublikum zunächst adressiert wird, um die private Kommunikationssituation brieflicher Korrespondenz simulierend einen gemeinsamen Horizont des Gesprächs zu konstituieren, mit dem ein wechselseitiges Verständnis und Vertrauen über die evozierte personale Wertschätzung hergestellt und eingefordert wird. Wenn Heine im ersten Abschnitt auf den „sehr lieben Brief“ seines Verlegers Dr. Heinrich Schultz antwortend, die Bedeutung Westfalens als Wiege Germaniens, respektive für die deutsche Nationwerdung am Beispiel von Armins Sieg über die Römer und der gewaltsamen Christianisierung der Sachsen durch Karl den Großen bis zur „biederen Ehrlichkeit“ und „anspruchslosen Tüchtigkeit“ der gegenwärtigen „Bewohner“ aufruft und damit dem erwachenden Selbstverständnis der Deutschen einerseits mit seinem schwärmerischen Erlebnisbericht über seinen Aufenthalt in Westfalen schmeichelt, um dieses hohe Lob der deutschen Provinz andererseits in der maßlosen Übertreibung ironisch $\mathrm{zu}$ brechen, so adaptiert er die Gattungspoetik des Privatbriefs nicht nur, sondern parodiert sie in ihrer floskelhaften Devotation zugleich:

Ihr sehr lieber Brief vom 5. D. M. hat mich mit der größten Freude erfüllt, da sich darin Ihr Wohlwollen gegen mich am unverkennbarsten aussprach. Es erquickt mir die Seele, wenn ich erfahre, daß so viele gute und wackere Menschen mit Interesse und Liebe meiner gedenken. [...] Es wird mir gewiß recht viel Vergnügen machen, wenn ich, wie Sie mir schreiben, durch Mitteilungen aus der Residenz mir so viele liebe Menschen verpflichte. ${ }^{10}$

Im Anschluss beendet er mit einer der gattungstypischen Authentifizierungsund Unmittelbarkeitsgesten, die die Definition des Briefs als Nachahmung eines persönlichen Gesprächs durch die unverzügliche zeitnahe Antwort zitieren, seine fingiert exklusive Adressierung des Briefeingangs: „Ich habe mir gleich bei Empfang Ihres Briefes Papier und Feder zurechtgelegt, und bin schon jetzt - am Schreiben." ${ }^{11}$ Der Leser des Rheinisch-Westfälischen Anzeigers darf sich in der Gewissheit wähnen, im Folgenden exklusiver stiller Teilhaber einer privaten Kommunikation zu werden: So wird er Zeuge zweier so unterschiedlicher gesellschaftlicher Ereignisse wie einer Prinzessinnenhochzeit, die Heine aus der Perspektive des einfachen Zuschauers /Lesers aus dem Volk beobachtet und des sich leitmotivisch wiederholenden Stellvertreterstreit zwischen der „deutschen“ und „italienischen“ Oper, mit dem Heine Kritik am politischen System Preußens nicht immer erfolgreich an der Zensur vorbei - in seinem launischen Kommentar zu üben versucht:

Der heftige Partheykampf von Liberalen und Ultras, wie wir ihn in andern Hauptstädten sehen, kann bei uns nicht zum Durchbruch kommen, weil die königliche Macht, kräftig und partheylos schlichtend, in der Mitte steht. Aber dafür sehen wir in Berlin oft einen ergötzlichern Partheykampf, den in der Musik. ${ }^{12}$

10. Heine, Briefe aus Berlin I, S. 9.

11. Ibid.

12. Ibid., S. 24. 
Heine nimmt in diesem Stellvertreterkampf weder für den einen, den italienischen Hofmusiker Gaspare Spontini und Repräsentanten eines musikalischen Orientalismus noch für den anderen, den Protagonisten der Deutschtümelei, Carl Maria von Weber, Stellung, sondern entscheidet sich für einen marginalen Dritten, nämlich den heute nahezu vergessenen Komponisten jüdischer Herkunft Georg Abraham Schneider und seine Oper Aucassin und Nicolette. Dieser setzt dem imperialistischen Gestus von Spontinis pompöser Ausstattungsoper Olympia, die E.T.A. Hoffmann für die Berliner Uraufführung im Jahr 1821 aus dem Französischen Voltaires übersetzt hatte und in der der Held am Ende auf einem Elefanten auf die Bühne reitet, Bilder eines märchenhaften Orients aus Tausend und Einer Nacht, der bei Heine für einen poetischen ,zusammengeträumten' Orient steht, gegenüber:

Was mich betrifft, so amüsierte mich diese Oper ganz außenordentlich. Mich erheiterte das bunte Mährchen, [...] mich ergötzte der anmuthige Kontrast vom ernsten Abendlande und dem heitern Orient. ${ }^{13}$

Mit diesem paradiesischen biblischen Orient, der bei Schneider den Hintergrund für den aus dem französischen Mittelalter stammenden Stoff einer christlichmoslemischen Liebesgeschichte bildet und für Heine Schauplatz des Beginns der Menschheitsgeschichte wie Heimat des Judentums zugleich ist, identifiziert sich Heine. Diesen poetisch überhöhten Orient konfrontiert Heine in seiner politischen Polemik mit der gegenläufigen, regressiven Geschichte des gegenwärtigen Okzidents in der Gestalt Preußens: Dem kunstvollen Gesang der Kanarienvögel „in den Thälern Kaschimirs“ wie unserer „Ureltern“14 aus der Frühgeschichte der Menschheit stehen in Preußen „bellende Hunde“, „grunzende Schweine“, „brummende Bären“ oder gar „Esel“ und „Hammel“ gegenüber, so Heines Kulturvergleich von Orient und Okzident. Diesen historischen Orient als Raum früher Hochkulturen konfrontiert Heine mit Spontinis exotistischem Orientalismus in Gestalt seiner neuesten, für die Hochzeitsfeierlichkeiten der Prinzessin Alexandrine komponierten Oper Nurmahal, oder das Rosenfest in Kaschemir, über die das Gerücht geht, dass sie mit ihrer effekthascherischen Inszenierung eines modischen Orientalismus den exquisiten Geschmack des verwöhnten höfischen Publikums zu befriedigen versuche:

Daß zwei Elephanten im Rosenfest vorkommen würden, hatte ich wirklich selbst gehört. Nachher sagte man mir, es wären nur zwey Kameele, später hieß es, zwey Studenten kämen drin vor, und endlich sollten es Unschuldsengel seyn. ${ }^{15}$

Wie später Else Lasker-Schüler operiert Heine hier mit zwei entgegengesetzten Orient-Diskursen, einem jüdischen der kulturellen Selbstbeschreibung zwischen ,orientalischem' und ,okzidentalem' Judentum und einem eurozentrischen Orientalismus der exkludierenden Erfindung des Anderen. So spielt er einen poetischen Orient als Raum hybrider jüdisch-islamischer Hochkultur in der

13. Ibid., S. 27.

14. Ibid.

15. Ibid., S. 42. 
Tradition der Aufklärung Lessings ${ }^{16}$, dessen Geist Heine im Stadtbild Berlins vergegenwärtigt, gegen die Inszenierungen eines preußischen Orientalismus zur Herrschaftsrepräsentation aus, wenn er den Kutscher des Herzogs von Cumberland in seiner phantastischen Maskerade orientalischer Métissage mit seiner plastischen Schilderung der Lächerlichkeit preisgibt:

\begin{abstract}
Was ist Salomo in seiner Königspracht, was ist Harun-al-Raschid in seinem Kalifenschmuck, ja was ist der Triumphelephant in der Olympia gegen die Herrlichkeit dieses Herrlichen? [...] Aber heute trug er ein karmoisinrothes Kleid, das halb Frack, halb Ueberrock war, Hosen von derselben Farbe, alles mit breiten goldnen Tressen besetzt. Sein edles Haupt, kreideweiß gepudert, und mit einem unmenschlich großen schwarzen Haarbeutel gezirt, war von einem schwarzen Samtkäppchen mit langem Schirm bedeckt. [...] Aber Er trug die gewöhnliche Herrscherwürde im Antlitz, Er dirigirte die sechsspännige Staatskarosse. ${ }^{17}$
\end{abstract}

Abschließend kann festgehalten werden, dass sich Heine im Feuilleton der epistolaren Plauderei zur Camouflage seiner politischen Kritik an Preußen bedient, die er hinter seinen launisch-ironischen Beobachtungen des alltäglichen gesellschaftlichen Lebens in der Hauptstadt vor der Zensur versteckt. Der adressierte Leser dieser Briefe sollte und konnte ihre kulturkritische politische Botschaft zwischen den Zeilen scheinbar harmlos privater Plauderei lesen.

\title{
Alfred Kerrs Briefe aus Berlin 1897-1898
}

Alfred Kerr versteht seine Berliner Briefe für die Breslauer Zeitung, die er zwischen 1897 und 1900 schreibt, als Chroniken, Erzählungen und Plaudereien über das Hauptstadtleben. Auch er spricht immer wieder seinen Leser, unmittelbar auf den subjektiven und privaten Charakter von Mitteilungen in Briefform rekurrierend, an, allerdings bindet er ihn nicht direkt in einen fiktiven Dialog ein wie vor ihm Heine, sondern bedient sich als Adressierung an den ,geneigten' Leser eingeführter Abschiedsformeln wie „Adieu, Leser“, „Leben Sie wohl“, „Gute Nacht, Leser“ oder „Bleiben Sie gesund, Leser“, wenn er den Leser der Breslauer Zeitung als Kollektivsubjekt anspricht.

Darüber hinaus weist Kerr wie vor ihm schon Heine durch kritische Auseinandersetzungen mit anderen Korrespondenten und der Form des Korrespondentenberichts auf den Kunstcharakter des Briefs im Feuilleton hin. So stellt Kerr seine Briefe aus Berlin bewusst in die Tradition von Heine und Peter Altenberg ein, allerdings nicht ohne sich kritisch von beiden abzusetzen. Heines Briefe verharmlost er, ihren eminent kritischen Charakter verkennend, $\mathrm{zu}$ minderwertigen literarischen Produkten, den Schulterschluss zu seinem Intimfeind Karl Kraus und dessen Heine-Schelte suchend:

16. So ,plaudert' Heine im Ersten Brief: „Aber ich sehe, Sie hören schon nicht mehr, was ich erzähle, und staunen die Linden an. Ja, das sind die berühmten Linden, wo von Sie so viel gehört haben. Mich durchschauert's, wenn ich denke, auf dieser Stelle hat vielleicht Lessing gestanden“. Ibid., S. 13.

17. Ibid., S. 39 . 
Als unser lieber Heinrich Heine Berliner Briefe schrieb (sie gehören seltsamerweise zum Schlechtesten, was er hervorbrachte), hatte er es gut. Die Vermählung irgendeiner preußischen Alexandrine mit irgendeinem mecklenburgischen Prinzen konnte hinreichen, einen Berliner Brief zu verfassen. ${ }^{18}$

In der Moderne, in der das Leben „zerflattert und zerstiebt“, diagnostiziert Kerr dagegen den Verlust von Einheit, die er bei Heine noch im Preußentum zu erkennen glaubt. Kerr versteht sich dagegen als „getreuer Chronist“ eines „dezentralisierten“ Großstadtlebens, das er zu zersplitterten „Mosaikbildern“19 künstlerisch wieder zusammenfügen möchte. Als dieser schreibt er denn auch über ein zeittypisches Phänomen des kulturellen Großstadtlebens, das alle Feuilletons der Zeit beherrscht, die Völkerschauen und Orientausstellungen auf den Gewerbeausstellungen, in den Vergnügungsparks und Panoptiken. Als Abfallprodukte des europäischen Kolonialismus befriedigen diese kommerziellen Ausstellungen ein Massenbedürfnis nach Exotismus unter dem Vorwand, Wissen aus der den Kolonialismus flankierenden Wissenschaft der Völkerkunde über ,das Fremde' und ,den Fremden' populär zu vermitteln. Dabei weist Kerr kritisch auf den Inszenierungscharakter der Konstruktionen des Fremden in den Völkerschauen am Beispiel der Kulissenstadt „Kairo“ auf der Berliner Gewerbeausstellung von 1896 hin, in der dem Zuschauer all das geboten wird, was seine Erwartungen in Bezug auf das ,Fremde', einschließlich dem nach Authentizität des Darstellten, bedient:

[..] im Grunde ist ,Kairo' nur ein enormes Tingeltangel. [...] der ganze seltsame Zauber morgenländischer Pracht tritt bannend zutage, [...] dort tanz ein Derwisch den grausigen Muscheltanz, in dem er wie der Gottseibeiuns klappert und heult, dort jagt eine Schöne mit schwarzem Teint auf einem Schimmel dahin, hier ladet ein Türke zu einer Tasse Kaffee ein, hier ist ein verschwiegener Raum, in dem man den berauschenden Bauchtanz vorführt - [...] und all diese östlichen Männer und Weiber, von der gelben bis zur tiefschwarzen Gesichtsfarbe, sind vom Orient unmittelbar nach Berlin transportiert worden. Sie sind sich der Schaustellung, die ihr Amt ist, wohl bewußt und posieren wahrscheinlich grenzenlos. ${ }^{20}$

Seine Kritik an dem Rassismus dieser Völkerschauen, in denen sich der politische Herrschaftsanspruch der Kolonialherren als Definitionshoheit über den Fremden unverhohlen bloß stellt, macht Kerr durch die Umkehrung des Beobachterstatus und damit den von Subjekt und Objekt der Betrachtung deutlich, wenn er den Anderen einen befremdlichen Blick auf die deutsche Kultur zurückwerfen lässt:

Alles in allem wird die indische Ausstellung mehr ,machen' als das verkrachte Transvaal; aber weniger als Venedig aus Pappe, weniger als Kairo in Treptow, und im nächsten Jahr wird vielleicht Lappland auf ihren Trümmern erstehn, denn die Völker der Erde marschieren der Reihe nach am Kurfürstendamm auf und machen sich dabei ethnologische Notizen über die Berliner. Sie werden da aufschreiben, daß es ein Volk von jungen Leuten ist, welche unbegründet anfangen zu blöken, kille-kille zu rufen, zu drängeln; ein Volk, dessen junge Mädchen oft durch Biergenuß beschwipst sind und den Hut schief aufhaben; und ein Volk,

18. Kerr, Wo liegt Berlin?, S. 80.

19. Ibid.

20. Ibid., S. 152. 
dessen Nationalhymne mit den Worten beginnt: ,Es war ein Sonntag hell und klar. Manche behaupten, sie beginne: „Emma, mein geliebtes Mauseschwänzchen.'21

Trotz dieser Kritik am institutionalisierten Orientalismus in der Hauptstadt des späten Kaiserreichs bedient sich Kerr in seinen Briefen des im Feuilleton eingeführten gefälligen, überwiegend harmlosen Plaudertons, über den Karl Kraus in seinem Essay Heine und die Folgen sein vernichtendes Urteil gesprochen hatte. So schränkt er seine Orientalismuskritik, um seinem Breslauer Publikum nicht $\mathrm{zu}$ nahe $\mathrm{zu}$ treten und den Lesegenuss $\mathrm{zu}$ verderben, unmittelbar mit der rhetorischen Figur des „sowohl als auch“ oder durch Formulierungen wie diese immer wieder ein: „Es ist ja wahr: im Grunde ist „Kairo’ nur ein enormes Tingeltangel. Aber eines, das die Phantasie in ungeahntem Maße anregt. Hier ist der leibhafte Orient.“ Abschließend kommt er zu einem alle Kritik nivellierenden Urteil: „Das Ganze ist, wie angedeutet, ein starker Mumpitz - aber doch unleugbar ein sehr geistvoller und ein sehr anregender Mumpitz." ${ }^{22}$

Am Ende des Jahrhunderts ist aus Heines exklusivem Berliner Orientalismus für die geschlossene Gesellschaft des preußischen Hofs ein Phänomen für die Unterhaltung der einheimischen Massen und des frühen Tourismus' geworden, bei dem der Reisende die „Fremde“ im eigenen Land bestaunen kann. Im Juni 1896 wartet ,Kairo', so weiß der Korrespondent zu berichten, mit einer Touristenattraktion auf, die wohlfeile Fremdheitserfahrungen für jedermann bei einem opulent gestalteten „orientalischen Nachtfest“ offeriert. Wiederum schwärmt Kerr in höchsten Tönen von diesem frühen, aufwendig inszenierten Event der Tourismusindustrie:

Nun hat die Direktion von „Kairo“ etwas Besonderes für die Fremden tun wollen und am Freitag ein orientalisches Nachtfest veranstaltet. Es war in Wahrheit ungewöhnlich hübsch. Ganz wundervoll orientalisch und dabei doch immer ein kleiner parodistischer Beigeschmack. ${ }^{23}$

Die theatralische Masseninszenierung verfehlt auch beim kritisch beobachtenden Korrespondenten ihre beabsichtigte Wirkung nicht, wenn sie einerseits die vertraute Welt durch den Aufbau einer gigantischen Kulisse aus exotischen Farben und Formen beeindruckt, ihren Zuschauer in fremde Welten entführt und orientalisch verzaubert, andererseits aber ungewollt auch etwas Unheimliches, Bedrohliches und Unheilvolles ausstrahlt. Die Ambivalenz dieser exotistischen Performance macht unter der Oberfläche dieses phantastischen Vergnügens eine latente Verunsicherung im Beobachter spürbar, die auf die Problematik dieses zweifelhaften Spektakels deutscher wie europäischer Großmachtpolitik hinweist:

Die große Pyramide, deren unbequem riesige Stufen sich massig vom Nachthimmel abhoben, glomm im roten Feuerschein, der von unten dämmerhaft und zitternd auf sie fiel. Und im Schatten der Steinstufen standen unbeweglich in ihren weißen Gewändern die Beduinen, die braunen Köpfe halb verhüllt, in den dunklen Augen zugleich Phlegma,

21. Ibid., S. $387 \mathrm{f}$.

22. Ibid., S. 152.

23. Ibid., S. 162. 
Müdigkeit und latentes Feuer. Unten der Rest der großen Schar, seltsam gruppiert mit allen Kamelen, Gazellen, Rossen und Eseln. Ein einziges Funkeln von Waffen und Steinen. Die weißen Gewänder leichteten durch die Nacht. Eine Riesengruppe voll schwermütiger Ruhe und zugleich zurückgehaltener wilder Bewegung. ${ }^{24}$

Gegenüber Heine erweist sich Kerr mit seinen untergründigen, impressionistischen Stimmungsbildern des vorsichtigen Andeutens und der ambivalenten Kippfiguren eher als leiser Prophet eines kommenden Unheils am Ende des Kaiserreichs denn als offener, kritischer Analytiker.

\section{Else Lasker Schülers Briefe nach Norwegen 1911}

Else Lasker-Schüler bezieht sich mit ihren Briefen nach Norwegen im Sturm kritisch auf die Berlin-Briefe ihres unmittelbaren Vorgängers und Zeitgenossen, indem sie mit der eingeführten Gattung ihr Spiel treibt und die Form im direkten Dialog mit Heine und Kerr parodiert: So kehrt Lasker-Schüler als erstes die Zielrichtung und die Adressierung ihrer Briefe um: Während Heine und Kerr aus der Hauptstadt in die Provinz berichten und damit Distanz und Fremdheit für den Leser abbauen wollen, berichtet Lasker-Schüler aus der vertrauten Metropole Berlin an kurzfristig Abwesende und darüber hinaus an ein überwiegend in Berlin ansässiges Publikum der expressionistischen Zeitschrift. Nicht Fremdbeobachtung, sondern Selbstbeobachtung betreiben LaskerSchülers Briefe, Subjekt und Objekt der Beobachtung werden hier untrennbar im Selbstexperiment verschmolzen, mehr noch; die Leser und Mitautoren der Zeitschrift liefern das Figurenpersonal für diese Briefe. Daher geht es in den Briefen nach Norwegen nicht darum, Distanz zu überwinden, sondern um eben das genaue Gegenteil, nämlich das Bekannte und Vertraute fremd werden zu lassen und damit die begonnene Umkehrung der Blickrichtung bei Kerr konsequent auf die Spitze zu treiben. Zudem richtet die Schreiberin ihren Blick nicht aus einer unbeteiligten Beobachterperspektive auf ein fremdes Großstadtgeschehen, sondern macht sich selbst und ihr unmittelbares Umfeld zu Protagonisten ihrer distanzlosen, verfremdenden Beobachtungen, indem sie zu der ursprünglichen Form des privaten Briefs zurückkehrt, wenn sie von scheinbar Intimstem, z. B. ihren vielen - meist unglücklich verlaufenden - Liebesaffären, offen plaudert. Darüber hinaus lässt sie ihre Freunde in den Briefen entweder gänzlich unverhüllt oder unter leicht zu dechiffrierenden Phantasienamen auftreten, so dass diese sich bei ihr aufgrund eines vermeintlichen Vertrauensbruchs beschweren und sich eine weitere Erwähnung in den Briefen verbitten, wie es Lasker-Schüler von Karl Kraus in ihren Briefen überliefert, der sich in einem Brief an Herwarth Walden verwahrt habe, weiterhin als „Dalai-Lama“ bezeichnet zu werden. Daraufhin veranlasst Lasker-Schüler in einem der nächsten Briefe den Herausgeber des Sturms zu einem öffentlichen Aufruf in seiner Zeitschrift: 
Lieber Herwarth, willst Du im Sturm veröffentlichen lassen, daß sich alle Vertreter unseres gemeinschaftlichen Cafés melden mögen, die den Wunsch hegen, nicht mehr in den Briefen an Euch erwähnt zu werden. Ich gewähre ihnen freien Abzug. ${ }^{25}$

Über eine Beschwerde ihrer Haushaltshilfe Grete, die durch eine Erwähnung in den Briefen „ihr Ehrgefühl angegriffen“ ${ }^{26}$ und ihre Heiratspläne schwinden sieht, weiß die Briefschreiberin in einem anderen Brief zu berichten und fürchtet daher die Folgen einer Kündigung der treuen Angestellten. Schließlich bittet sie in einem weiteren Brief an den Adressaten und Juristen Kurt Neimann, der Herwarth Walden auf die Norwegenreise mitgenommen hatte, scherzhaft um rechtlichen Beistand in Bezug auf eine vorauseilende „Unterlassungsklage“ eines „Urenkels Bachs“, der bei einer möglichen Erwähnung in den Briefen mit seinem Selbstmord drohe:

Ferner will sich ein Urenkel Bachs das Leben nehmen, [...] falls ich ihn erwähne in meiner Korrespondenz. [...]

Lieber Kurt. Er drohte mir gestern selbst. Ist meine Antwort juristisch einwandsfrei? Mein Herr. Sie wollen sich das Leben nehmen, falls ich Sie im Sturm erwähne, oder haben Sie vor mich indirekt auf die Idee zu bringen? [...] Aber bis jetzt kämen Sie für mich noch nicht als Modell in Frage weder als Portrait noch als Karikatur. Zwar ist es mir schon gelungen aus einer prüden Null ein Wort zu formen. Aber gedulden Sie sich, seinen Sie guten Mutes. Hochachtungsvoll. ${ }^{27}$

Auf diese Weise kommentiert und reflektiert die Briefschreiberin nicht nur ihr ungewöhnliches poetisches Projekt, sondern macht darüber hinaus die Wirkung ihrer Briefe selbst zum Gegenstand weiterer epistolarer Einlassungen und Auseinandersetzungen, so dass sie den dialogischen Charakter epistolarer Kommunikation in der literarischen Fiktion einholt.

Else Lasker-Schülers Briefe nach Norwegen parodieren so die Form des privaten Briefs im Sturm, indem sie die Leser zu Voyeuren einer vermeintlich privat-intimen Korrespondenz mit dem Ex-Ehemann und Herausgeber der Zeitschrift, Herwarth Walden, und seinem Freund machen. So adressiert sie ihre Briefe unmittelbar - humorvoll bis ironisch - an diese beiden unter ständig wechselnden Anreden wie „Liebe Jungens“, „liebe Brüder“ oder „Kinder“, „Ihr lieben Freunde“, „Liebe Reisende“, „Liebe Nordländer“, Liebe Skiläufer“ und „Nordpolfahrer“, „liebe Eiskühler“ oder sogar „Liebe Renntiere“ oder einfach nur „Liebe Beide“ oder im letzten Brief „Liebe Gesandte“, als die Adressaten längst wieder zurück in Berlin sind.

Gleichzeitig setzt sie sich kritisch mit dem Brief als einer genuin literarischen Gattung des unmittelbaren authentischen Selbstausdrucks im dialogischen Bezug auf einen anderen auseinander. Denn sie treibt in ihren Briefen ein verwirrendes Spiel mit unterschiedlichen Maskeraden, indem sie Lesererwartungen an einen authentischen Selbstentwurf eines mit sich selbst identischen Individuums nicht nur enttäuscht, sondern auch konterkariert. Als weibliches Subjekt entwirft sich

25. Else Lasker-Schüler, „Briefe nach Norwegen“, in Der Sturm 2 (Dezember 1911), Nr. 88, S. 702.

26. Ibid., Nr. 90, S. 718.

27. Ibid., Nr. 89, S. 710. 
die Briefschreiberin in vielfältigen Rollen und Maskeraden, die in der Forschung weitgehend beschrieben worden sind ${ }^{28}$ : Neben der Rolle der modernen hysterischen Großstädterin mit ihren Liebes- und Todesspielen im schnellen Stimmungswechsel zwischen „himmelhoch jauchzend, zu Tode betrübt“ oder der des Clowns oder der dummen Augustine ragt ihre transsexuelle Inszenierung als orientalischer Prinz heraus, mit der sie ihre eigenen literarischen Texte, die Nächte der Tino von Bagdad und den Prinz Jussuf von Theben zitiert und in die epistolare Gattung einschmuggelt, um sie dort fortzuschreiben. Mit dieser Maskerade als orientalischer Märchenprinz leistet sie gleichzeitig dreierlei: Erstens knüpft sie an Heines doppelten Orientdiskurs aus seinen Berliner Briefen an, zweitens formuliert sie in dieser Maskerade ihre Kritik am zeittypischen Orientalismus und drittens identifiziert sie sich in dieser Maske mit den marginalisierten Fremden. Hatte Heine sich in seinen Briefen selbst in einer Position des Nicht-Identischen, der Differenz, zwischen Orient und Okzident wie in seiner späteren Lyrik ${ }^{29}$ entworfen, so greift Else Lasker-Schüler diesen literarischen Selbstentwurf Heines in der Rolle des Prinz von Theben in den Briefen wieder auf.

Den eng mit dem deutschen Kolonialismus verbundenen Orientalismus entzaubert die Briefschreiberin durch ihre grotesken Verfremdungen und ihre Verwirrung der Diskursordnung, indem sie den phantastischen Orient der OrientAusstellungen in ihren Erzählungen wörtlich nimmt und bei ihrem Besuch in der Ägyptenausstellung im Lunapark Realität werden lässt. Wenn Kerr den Akteuren noch den Vorwurf machte, inauthentisch zu sein, nicht ,Natur', sondern Kultur zu repräsentieren, da auch sie wie ihre Aussteller aus Geschäftssinn handelten, um das koloniale Phantasma der Betrachter zu spiegeln und zu befestigen, wechselt Else Lasker-Schüler die Beobachterposition, indem sie sich mit den Objekten der Ausstellung im entfesselten Tanz verbrüdert. Damit reißt sie die Grenze zwischen Fremdem und Eigenem, zwischen Objekt und Subjekt der Beobachtung, zwischen Phantasma und Lebenswelt ein, indem sie Kerrs Würdigung der Ausstellung als „phantasieanregend“ wörtlich nimmt, wenn sie ihren Brief über einen Besuch im Lunapark gleichzeitig auch zu einem Produkt der Phantasie auf zweiter Ebene, zur literarischen Umschrift von Kerrs Feuilleton macht:

Aber bei den Bauchtänzerinnen ereignete sich eines der Wunder meines arabischen Buches; ich tanzte mit Minn, dem Sohn des Sultans von Marokko. Wir tanzten, tanzten wie zwei Tanzschlangen, oben auf der Islambühne, wir krochen ganz aus uns heraus, nach den Locktönen der Bambusflöte des Bändigers nach der Trommel, pharaonenalt, mit den ewigen Schellen. ${ }^{30}$

28. Sylke Kirschnick, Tausend und ein Zeichen. Else Lasker-Schülers Orient und die Berliner Alltagsund Populärkultur um 1900, Würzburg, Königshausen \& Neumann, 2007.

29. Vgl. dazu Kathrin Wittler, die am Beispiel ihrer diskursanalytischen Lektüre des Gedichts „Jehuda ben Halevy“ von Heines ,jüdischer Differenzpoetik des Westöstlichen“ spricht und betont, dass er sich „einer eindeutigen Festlegung des Jüdischen auf Orient oder Okzident entzieht, den Grenzbereich aber umso präsenter hält.“ Kathrin Wittler, „,Mein westöstlicher dunkler Spleen: Deutsch-jüdische Orientimaginationen in Heinrich Heines ,Jehuda ben Halevy““, in Heine-Jahrbuch, 49. Jg., 2010, S. 31-49, hier S. 44.

30. Lasker-Schüler, „Briefe nach Norwegen“, in Der Sturm 2 (September 1911), Nr. 77, S. 615 f. 
Von der Verbrüderung mit den Fremden aus dem Lunapark bis zur Übernahme der Rolle des Prinz Jussuf und zur Orientalisierung Berlins zum ägyptischen Theben der israelitischen Gefangenschaft ist es nur noch ein kleiner Schritt in den Briefen. Ihre letzten Briefe unterschreibt die Korrespondentin daher konsequent mit „Euer Prinz von Theben“, der in seiner preußisch-orientalischen Hauptstadt als Herrscher gefeiert wird:

Telegramm.

Eben regierender Prinz in Theben geworden. Es lebe die Hauptstadt und mein Volk!!

Ich hoffe, Dich haben meine Briefe nicht gelangweilt, oder hat Kurtchen oft gegähnt? [...] Ich habe mir nie ein System gemacht, wie es kluge Frauen tun, nie eine Weltanschauung mir irgendwo befestigt, wie es noch klügere Männer tun, nicht einmal eine Arche habe ich mir gezimmert. Ich bin ungebunden, überall liegt ein Wort von mir, von überall kam ein Wort von mir, ich empfing und kehrte ein, so war ich ja immer der regierende Prinz von Theben. Wie alt bin ich, Herwarth? Tausend und vierzehn. Ein Spießbürger wird nie tausend und vierzehn. ${ }^{31}$

Else Lasker-Schülers Briefe nach Norwegen treiben ein karnevaleskes Spiel mit der eingeführten Gattung des Briefs, indem sie Gattungsgrenzen und -normen verletzt: Sie bilden so eine hybride Form aus dem Korrespondentenbericht im Feuilleton, dem privaten Brief als Ego-Dokument und dem literarischen Brief. Diese Grenzüberschreitungen funktionieren in beide Richtungen, indem sie nicht nur den als faktuale Erzählung konzipierten Korrespondentenbericht durch phantastische Erzählungen fiktionalisieren, sondern auch reale Personen und Ereignisse aus ihrem Umfeld zum Gegenstand ihrer poetischen Erfindungen machen. So verstößt sie mit ihren öffentlichen Selbstinszenierungen in ihren fingierten Briefen gegen Regeln von Privatheit, Authentizität und Wahrhaftigkeit, wenn sie scheinbar Privates und Intimes ausplaudert und Personen des Künstlerkreises um den Sturm in fiktionale Narrationen verstrickt, die die Grenzen der autobiographischen Gattung des Privatbriefs zur Autofiktion entgrenzt und den öffentlichen Brief als Korrespondentenbericht poetisiert. Gleichzeitig nutzt sie den privaten, an eine exklusive Adresse gerichteten Brief um scheinbar Intimstes ausplaudernd, private Kommunikationssituationen zu simulieren und das eigene Leben wie das ihrer Künstlerfreunde poetisch zu verfremden. Darüber hinaus unterläuft sie durch ein verwirrendes Spiel mit unterschiedlichsten Maskeraden eine zentrale Erwartung an Ego-Dokumente wie den Brief, nämlich seinem Leser einen unmittelbaren und authentischen Selbstausdruck des schreibenden Subjekts im dialogischen Bezug auf seinen Adressaten zu vermitteln.

Heine, Kerr und Lasker-Schüler beobachten mit ihren Berliner Briefen ihre Gegenwart und leisten einen Beitrag zu einem kulturkritischen Politikdiskurs, indem sie Diskurskritik wie am Beispiel des Orientalismus leisten, dem sie einen anderen Orientdiskurs störend gegenüberstellen. Gleichzeitig reflektieren und unterlaufen sie die autobiographische Gattung des Briefs als Camouflage ihrer Kritik in der scheinbar privat-subjektiven epistolaren Plauderei und reflektieren und subvertieren schließlich gängige identitätslogische Narrationen und Konzepte des Briefs als Ego-Dokument.

31. Ibid. S. 258-259. 
